\title{
p-Brane Solutions in Diverse Dimensions
}

\author{
I.Ya.Aref'eva $\stackrel{*}{,}$ K.S.Viswanathan ${ }^{\dagger}$ \\ and \\ I.V.Volovich $\ddagger$ \\ Department of Physics, Simon Fraser University \\ Burnaby, British Columbia, V5A 1S6, Canada
}

\begin{abstract}
A generic Lagrangian, in arbitrary spacetime dimension, describing the interaction of a graviton, a dilaton and two antisymmetric tensors is considered. An isotropic p-brane solution consisting of three blocks and depending on four parameters in the Lagrangian and two arbitrary harmonic functions is obtained. For specific values of parameters in the Lagrangian the solution may be identified with previously known superstring solutions.
\end{abstract}

*Permanent address: Steklov Mathematical Institute, Vavilov St.42, GSP-1, 117966, Moscow, e-mail: arefeva@class.mian.su

${ }^{\dagger}$ e-mail: kviswana@sfu.ca

${ }_{\ddagger}^{\ddagger}$ Permanent address: Steklov Mathematical Institute, Vavilov St.42, GSP-1, 117966, Moscow, e-mail: volovich@class.mian.su 


\section{Introduction}

Recent remarkable developments in superstring theory lead to the discovery that five known superstring theories in ten dimensions are related by duality transformations and that there are also $M$-theory in $11 d$ and $F$-theory in $12 d$ that are useful in the study of the moduli space of quantum vacua [1] - [6]. Duality requires the presence of extremal black holes in the superstring spectra. A derivation of the Bekenstein-Hawking formula for the entropy of certain extremal black holes was given using the D-brane approach [7]- 15]. In all these developments the study of p-brane solutions of the supergravity equations play an important role [16]-37].

In this paper we shall consider the following action

$$
I=\frac{1}{2 \kappa^{2}} \int d^{D} x \sqrt{-g}\left(R-\frac{1}{2}(\nabla \phi)^{2}-\frac{1}{2(q+1) !} e^{-\alpha \phi} F_{q+1}^{2}-\frac{1}{2(d+1) !} e^{\beta \phi} G_{d+1}^{2}\right),
$$

It describes the interaction of the gravitation field $g_{M N}$ with the dilaton $\phi$ and with two antisymmetric fields: $F_{q+1}$ is a closed $q+1$-differential form and $G_{d+1}$ is a closed $d+1$-differential form. Various supergravity theories contain the terms from (1.1).

The aim of this paper is to present a solution of (1.1) with the metric of the form

$$
d s^{2}=H_{1}^{-2 a_{1}} H_{2}^{-2 a_{2}} \eta_{\mu \nu} d y^{\mu} d y^{\nu}+H_{1}^{-2 b_{1}} H_{2}^{-2 a_{2}} \delta_{n m} d z^{n} d z^{m}+H_{1}^{-2 b_{1}} H_{2}^{-2 b_{2}} \delta_{\alpha \beta} d x^{\alpha} d x^{\beta},
$$

where $\eta_{\mu \nu}$ is a flat Minkowski metric,

$$
\mu, \nu=0, \ldots, q-1 ; \quad m, n=1,2, \ldots, d-q,
$$

and

$$
\alpha, \beta=1, \ldots, D-d
$$

For definitness we assume that $D>d \geq q$.

The parameters $a_{i}$ and $b_{i}$ in the solution (1.2) are rational functions of the parameters in the action (1.1):

$$
\begin{gathered}
a_{1}=\frac{2 \tilde{q}}{\alpha^{2}(D-2)+2 q \tilde{q}}, \quad a_{2}=\frac{\alpha^{2}(D-2)}{\alpha^{2} d(D-2)+2 \tilde{d} q^{2}} \\
b_{1}=-\frac{2 q}{\alpha^{2}(D-2)+2 q \tilde{q}}, \quad b_{2}=-\frac{\alpha^{2} d(D-2)}{\tilde{d}\left[\alpha^{2} d(D-2)+2 \tilde{d} q^{2}\right]}
\end{gathered}
$$

where

$$
\tilde{d}=D-d-2, \quad \tilde{q}=D-q-2 .
$$

Our solution (1.2) is valid only if the following relation between parameters in the action is satisfied

$$
\alpha \beta=\frac{2 q \tilde{d}}{D-2}
$$

There are two arbitrary harmonic functions $H_{1}$ and $H_{2}$ of variables $x^{\alpha}$ in (1.2),

$$
\Delta H_{1}=0, \quad \Delta H_{2}=0 .
$$

Non-vanishing components of the differential form are given by

$$
\mathcal{A}_{\mu_{1} \ldots \mu_{q}}=h \epsilon_{\mu_{1} \ldots \mu_{q}} H_{1}^{-1}, \quad F=d \mathcal{A},
$$




$$
\mathcal{B}_{I_{1} \ldots I_{d}}=k \epsilon_{I_{1} \ldots I_{d}} H_{2}^{-1}, \quad G=d \mathcal{B}, \quad I=0, \ldots d-1 .
$$

Here $\epsilon_{123 . ., q}=1, \epsilon_{123 \ldots d}=1$ and $h$ and $k$ are given by the formulae

$$
\begin{gathered}
h^{2}=\frac{4(D-2)}{\alpha^{2}(D-2)+2 q \tilde{q}}, \\
k^{2}=\frac{2 \alpha^{2}(D-2)^{2}}{\tilde{d}\left[\alpha^{2} d(D-2)+2 q^{2} \tilde{d}\right]},
\end{gathered}
$$

The dilaton field is

$$
\phi=\frac{1}{2} \beta k^{2} \ln H_{2}-\frac{1}{2} \alpha h^{2} \ln H_{1} .
$$

We obtain the solution (1.2) by reducing the Einstein equations to the system of algebraic equations. To this end we introduce a linear dependence between functions in the Ansatz (see below). The solution (1.2) consists of three blocks, the first block consists of variables $y$, another of variables $z$ and the other of variables $x$ and all functions depend only on $x$. We shall call it the three-block p-brane solution.

We shall consider also the following "dual" action

$$
\tilde{I}=\frac{1}{2 \kappa^{2}} \int d^{D} x \sqrt{-g}\left(R-\frac{1}{2}(\nabla \phi)^{2}-\frac{1}{2(q+1) !} e^{-\alpha \phi} F_{q+1}^{2}-\frac{1}{2(\tilde{d}+1) !} e^{\tilde{\beta} \phi} G_{\tilde{d}+1}^{2}\right),
$$

where $G_{\tilde{d}+1}$ is a closed $\tilde{d}+1$-differential form. If $\tilde{d}$ is related to $d$ by (1.5) and

$$
\tilde{\beta}=-\beta
$$

then the solution for the metric (1.2) with the differential form $F(1.8)$ and the dilaton $(1.12)$ is valid also for the action (1.13). An expression for the antisymmetric field $G$ will be different, namely

$$
G^{\alpha_{1} \ldots \alpha_{\tilde{d}+1}}=k H_{1}^{\sigma_{1}} H_{2}^{\sigma_{2}} \epsilon^{\alpha_{1} \ldots \alpha_{\tilde{d}+1} \beta} \partial_{\beta} H_{2}^{-1}
$$

here $\epsilon^{123 . . \tilde{d}+2}=1$ and

$$
\sigma_{1}=\frac{\alpha \beta h^{2}}{2}\left(1-\frac{1}{\tilde{d}}\right), \quad \sigma_{2}=\frac{\beta k^{2}}{2}\left(\frac{1}{\tilde{d}}-1\right)
$$

The three-block p-branes solution for the Lagrangian with one differential form for various dimensions of the space-time was found in 38. It contains previously known $\mathrm{D}=10$ case [35, 8]. Equations of motion for the case of one form corresponds to equation of motion for ansatz (1.2), (1.8) and (1.15) for the dual action (1.13) when $\alpha=\beta$ and $q=\tilde{d}$

Note that the metric (1.2) describes also the solution for the action with the form $F_{q+1}$ replaced by its dual $F_{\tilde{q}+1}$ with $\tilde{q}+q+2=D$ and $\alpha \rightarrow \tilde{\alpha}=-\alpha$. One can also change two forms $F$ and $G$ to their dual version without changing the metric (1.2).

To illustrate our method on a simple example we first consider in Sect. 3 the simple case when in (1.1) $d=q$ and one has only two blocks in the metric. Then in Sect. 4 we derive the solution (1.2). In Sect. 5 we consider particular cases of the solution (1.2) and obtain different known solutions. In Appendix the solution of the system of algebraic equations is given. 


\section{Two block solution}

To illustrate the method of solution in this section we consider the simple case when the system of algebraic equations can be easily solved. Let us consider the action

$$
I=\frac{1}{2 \kappa^{2}} \int d^{D} z \sqrt{-g}\left[R-\frac{1}{2}(\partial \phi)^{2}-\frac{e^{-\alpha \phi}}{2(d+1) !} F_{d+1}^{2}-\frac{e^{\beta \phi}}{2(d+1) !} G_{d+1}^{2}\right)
$$

The Einstein equations for the action (1.13) read

$$
R_{M N}-\frac{1}{2} g_{M N} R=T_{M N}
$$

where the energy-momentum tensor has the form

$$
\begin{gathered}
T_{M N}=\frac{1}{2}\left(\partial_{M} \phi \partial_{N} \phi-\frac{1}{2} g_{M N}(\partial \phi)^{2}\right) \\
+\frac{1}{2 d !} e^{-\alpha \phi}\left(F_{M M_{1} \ldots M_{d}} F_{N}^{M_{1} \ldots M_{d}}-\frac{1}{2(d+1)} g_{M N} F^{2}\right)+ \\
\frac{1}{2 d !} e^{\beta \phi}\left(G_{M M_{1} \ldots M_{d}} G_{N}^{M_{1} \ldots M_{d}}-\frac{1}{2(d+1)} g_{M N} G^{2}\right)
\end{gathered}
$$

and one has also equations of motion

$$
\begin{gathered}
\partial_{M}\left(\sqrt{-g} e^{-\alpha \phi} F^{M M_{1} \ldots M_{d}}\right)=0 \\
\partial_{M}\left(\sqrt{-g} e^{\beta \phi} G^{M M_{1} \ldots M_{d}}\right)=0 \\
\partial_{M}\left(\sqrt{-g} g^{M N} \partial_{N} \phi\right)+\frac{\alpha}{2(d+1) !} \sqrt{-g} e^{-\alpha \phi} F^{2}-\frac{\beta}{2(d+1) !} \sqrt{-g} e^{\beta \phi} G^{2}=0
\end{gathered}
$$

We use the following Ansatz

$$
\begin{gathered}
F=d \mathcal{A}, \quad \mathcal{A}_{01 \ldots d-1}=\gamma_{1} e^{C_{1}(x)} \\
G=d \mathcal{B}, \quad \mathcal{B}_{01 \ldots d-1}=\gamma_{2} e^{C_{2}(x)} \\
d s^{2}=e^{2 A(x)} \eta_{\alpha \beta} d y^{\alpha} d y^{\beta}+e^{2 B(x)} d x^{i} d x^{i},
\end{gathered}
$$

$\alpha, \beta=0,1, \ldots, \mathrm{d}-1, \eta_{\alpha \beta}$ is a flat Minkowski metric, $i, j=\mathrm{d}, \ldots, \mathrm{D}$.

With the above Ansatz equations (2.18) are reduced to the following system of equations:

$$
\begin{aligned}
(d-1) \Delta A+(\tilde{d}+1) \Delta B+ & \frac{d(d-1)}{2}(\partial A)^{2}+\frac{\tilde{d}(\tilde{d}+1)}{2}(\partial B)^{2}+(d-1) \tilde{d}(\partial A \partial B)= \\
& -\frac{\gamma_{1}^{2}}{4} e^{\alpha \phi-2 d A+2 c_{1}}\left(\partial c_{1}\right)^{2}-\frac{\gamma_{2}^{2}}{4} e^{\alpha_{2} \phi-2 d A+2 c_{2}}\left(\partial c_{1}\right)^{2}-\frac{1}{4}(\partial \phi)^{2}(2.26)
\end{aligned}
$$


and

$$
\begin{gathered}
-d\left(\partial_{m} \partial_{n} A+\partial_{m} A \partial_{n} A\right)-\tilde{d}\left(\partial_{m} \partial_{n} B-\partial_{m} B \partial_{n} B\right)+d\left(\partial_{m} A \partial_{n} B+\partial_{m} B \partial_{n} A\right) \\
+\delta_{m n}\left[d \Delta A+\frac{d(d+1)}{2}(\partial A)^{2}+\tilde{d} \Delta B+\frac{\tilde{d}(\tilde{d}+1)}{2}(\partial B)^{2}+d(\tilde{d}-1)(\partial A \partial B)\right]= \\
\frac{1}{2}\left[\partial_{m} \phi \partial_{n} \phi-\frac{1}{2} \delta_{m n}(\partial \phi)^{2}\right]-\frac{\gamma_{1}^{2}}{4} e^{\alpha \phi-2 d A+2 C_{1}}\left[\partial_{m} C_{1} \partial_{n} C_{1}-\frac{1}{2} \delta_{m n}\left(\partial C_{1}\right)^{2}\right] \\
-\frac{\gamma_{2}^{2}}{4} e^{-\beta \phi-2 d A+2 C_{2}}\left[-\partial_{m} C_{2} \partial_{n} C_{2}-\frac{1}{2} \delta_{m n}\left(\partial C_{2}\right)^{2}\right]
\end{gathered}
$$

Here $\tilde{d}=D-d-2$. Equations for antisymmetric fields are

$$
\begin{gathered}
\partial_{m}\left(e^{-\alpha \phi-d A+\tilde{d} B+c_{1}} \partial_{m} c_{1}\right)=0 \\
\partial_{m}\left(e^{\beta \phi-d A+\tilde{d} B+c_{1}} \partial_{m} c_{2}\right)=0
\end{gathered}
$$

Equation of motion for dilaton

$$
\begin{gathered}
\partial_{m}\left(e^{-d A+\tilde{d} B} \partial_{m} \phi\right)-\frac{\alpha \gamma_{1}^{2}}{2} e^{-\alpha \phi-2 d A+2 C_{1}}\left(\partial_{m} C_{1}\right)^{2} \\
+\frac{\beta \gamma_{1}^{2}}{2} e^{\beta \phi-2 d A+2 C_{2}}\left(\partial_{m} C_{2}\right)^{2}=0
\end{gathered}
$$

We impose the following relations:

$$
\begin{gathered}
d A+\tilde{d} B=0, \\
-\alpha \phi-2 d A+2 C_{1}=0, \\
\beta \phi-2 d A+2 C_{2}=0 .
\end{gathered}
$$

Under these conditions equations (2.28), (2.29) and (2.30) take the following forms, respectively,

$$
\begin{gathered}
\partial_{m}\left(e^{-C_{1}} \partial_{m} C_{1}\right)=0, \\
\partial_{m}\left(e^{-C_{2}} \partial_{m} C_{2}\right)=0, \\
\Delta \phi-\frac{\alpha \gamma_{1}^{2}}{2}\left(\partial_{m} C_{1}\right)^{2}+\frac{\beta \gamma_{2}^{2}}{2}\left(\partial_{m} C_{2}\right)^{2}=0
\end{gathered}
$$

¿From equations (2.34), 2.35) and (2.36) we get

$$
\Delta C_{1}=\left(\partial C_{1}\right)^{2}
$$




$$
\Delta C_{2}=\left(\partial C_{2}\right)^{2}
$$

and

$$
\phi=\varphi_{1} C_{1}+\varphi_{2} C_{2}
$$

with

$$
\begin{gathered}
\varphi_{1}=\frac{\alpha \gamma^{2}}{2} \\
\varphi_{2}=-\frac{\beta \gamma^{2}}{2}
\end{gathered}
$$

Equations (2.32) and (2.33) give

$$
A=a_{1} C_{1}+a_{2} C_{2}
$$

where

$$
a_{1}=\frac{\beta}{d(\alpha+\beta)}, \quad a_{2}=\frac{\alpha}{d(\alpha+\beta)}
$$

and

$$
\phi=\varphi_{1} C_{1}+\varphi_{2} C_{2}
$$

where

$$
\begin{aligned}
& \varphi_{1}=\frac{2}{\alpha+\beta}, \\
& \varphi_{2}=-\frac{2}{\alpha+\beta} .
\end{aligned}
$$

Comparing (2.40) with (2.45) and (2.41) with (2.46) we conclude that

$$
\gamma_{1}^{2}=\frac{4}{\alpha(\alpha+\beta)}, \quad \gamma_{2}^{2}=\frac{4}{\beta(\alpha+\beta)} .
$$

Let us now consider equation (2.27). Since $C_{1}$ and $C_{2}$ are two independent functions we have to have that the terms with $\partial_{m} C_{1} \partial_{n} C_{2}$ vanish, i.e. we have to impose the condition

$$
\alpha \beta=\frac{2 d \tilde{d}}{d+\tilde{d}} .
$$

Therefore, the Ansatz (2.24) is consistent with the metric (2.25) only under condition (2.48). Straitforward calculations show that for $A$, and $\phi$ given by (2.42)-(2.43) and (2.44) in terms of two independent functions $C_{1}$ and $C_{2}$, satisfying equations (2.37) and (2.38), equations (2.26) (2.27) are satisfied if we impose the condition (2.48).

Let us write the metric in terms of two harmonic functions:

$$
H_{1}=-\ln C_{1}, \quad H_{2}=-\ln C_{2}
$$

We finally get

$$
\begin{aligned}
d s^{2}= & H_{1}^{-\frac{2 \beta}{d(\alpha+\beta)}} H_{2}^{-\frac{2 \alpha}{d(\alpha+\beta)}} \eta_{\alpha \beta} d y^{\alpha} d y^{\beta} \\
& +H_{1}^{\frac{2 \beta}{\overline{d(\alpha+\beta)}}} H_{2}^{\frac{2 \alpha}{d(\alpha+\beta)}} d x^{i} d x^{i}
\end{aligned}
$$




\section{Three block solution}

In this secction we consider equations of motion for the action (1.13). The Einstein equations for the action (1.13) read

$$
R_{M N}-\frac{1}{2} g_{M N} R=T_{M N}
$$

where the energy-momentum tensor is

$$
\begin{gathered}
T_{M N}=\frac{1}{2}\left(\partial_{M} \phi \partial_{N} \phi-\frac{1}{2} g_{M N}(\partial \phi)^{2}\right) \\
+\frac{1}{2 q !} e^{-\alpha \phi}\left(F_{M M_{1} \ldots M_{q}} F_{N}^{M_{1} \ldots M_{q}}-\frac{1}{2(q+1)} g_{M N} F^{2}\right) \\
+\frac{1}{2 \tilde{d} !} e^{-\alpha \phi}\left(G_{M M_{1} \ldots M_{\tilde{d}}} G_{N}^{M_{1} \ldots M_{\tilde{d}}}-\frac{1}{2(\tilde{d}+1)} g_{M N} G^{2}\right)
\end{gathered}
$$

The equation of motion for the antisymmetric fields are

$$
\begin{aligned}
& \partial_{M}\left(\sqrt{-g} e^{-\alpha \phi} F^{M M_{1} \ldots M_{q}}\right)=0, \\
& \partial_{M}\left(\sqrt{-g} e^{-\beta \phi} G^{M M_{1} \ldots M_{\tilde{d}}}\right)=0,
\end{aligned}
$$

and one has the Bianchi identity

$$
\begin{aligned}
\epsilon^{M_{1} \ldots M_{q+2}} \partial_{M_{1}} F_{M_{2} \ldots M_{q+2}} & =0 . \\
\epsilon^{M_{1} \ldots M_{\tilde{d}+2}} \partial_{M_{1}} G_{M_{2} \ldots M_{\tilde{d}+2}} & =0 .
\end{aligned}
$$

The equation of motion for the dilaton is

$$
\partial_{M}\left(\sqrt{-g} g^{M N} \partial_{N} \phi\right)+\frac{\alpha}{2(q+1) !} \sqrt{-g} e^{-\alpha \phi} F^{2}+\frac{\beta}{2(\tilde{d}+1) !} \sqrt{-g} e^{-\beta \phi} G^{2}=0 .
$$

We shall solve equations (3.1)-(3.7) by using the following Ansatz for the metric

$$
d s^{2}=e^{2 A(x)} \eta_{\mu \nu} d y^{\mu} d y^{\nu}+e^{2 F(x)} \delta_{n m} d z^{n} d z^{m}+e^{2 B(x)} \delta_{\alpha \beta} d x^{\alpha} d x^{\beta},
$$

where $\mu, \nu=0, \ldots, \mathrm{q}-1, \eta_{\mu \nu}$ is a flat Minkowski metric, $m, n=1,2, \ldots, r$ and $\alpha, \beta=1, \ldots, \tilde{d}+2$. Here $A, B$ and $C$ are functions on $x ; \delta_{n m}$ and $\delta_{\alpha \beta}$ are Kronecker symbols.

Non-vanishing components of the differential forms are

$$
\begin{gathered}
\mathcal{A}_{\mu_{1} \ldots \mu_{q}}=h \epsilon_{\mu_{1} \ldots \mu_{q}} e^{C(x)}, F=d \mathcal{A} \\
G^{\alpha_{1} \ldots \alpha_{\tilde{d}+1}}=\frac{1}{\sqrt{-g}} k e^{\beta \phi} \epsilon^{\alpha_{1} \ldots \alpha_{\tilde{d}+1} \gamma} \partial_{\gamma} e^{\chi},
\end{gathered}
$$

where $h$ and $k$ are constants. The left hand side of the Einstein equations for the metric (3.8) read

$$
R_{\mu \nu}-\frac{1}{2} g_{\mu \nu} R=\eta_{\mu \nu} e^{2(A-B)}[(q-1) \Delta A+(\tilde{d}+1) \Delta B+r \Delta F
$$




$$
\begin{aligned}
& +\frac{q(q-1)}{2}(\partial A)^{2}+\frac{r(r+1)}{2}(\partial F)^{2}+\frac{\tilde{d}(\tilde{d}+1)}{2}(\partial B)^{2} \\
+ & \tilde{d}(q-1)(\partial A \partial B)+r(q-1)(\partial A \partial F)+r \tilde{d}(\partial F \partial B)] \\
R_{m n}- & \frac{1}{2} g_{m n} R=\delta_{m n} e^{2(F-B)}[q \Delta A+(\tilde{d}+1) \Delta B+(r-1) \Delta F \\
+ & \frac{q(q+1)}{2}(\partial A)^{2}+\frac{r(r-1)}{2}(\partial F)^{2}+\frac{\tilde{d}(\tilde{d}+1)}{2}(\partial B)^{2} \\
+ & \tilde{d} q(\partial A \partial B)+q(r-1)(\partial A \partial F)+\tilde{d}(r-1)(\partial F \partial B)] \\
& R_{\alpha \beta}-\frac{1}{2} g_{\alpha \beta} R=-q \partial_{\alpha} \partial_{\beta} A-\tilde{d} \partial_{\alpha} \partial_{\beta} B-r \partial_{\alpha} \partial_{\beta} F \\
& -q \partial_{\alpha} A \partial_{\beta} A+\tilde{d} \partial_{\alpha} B \partial_{\beta} B-r \partial_{\alpha} F \partial_{\beta} F \\
+ & q(\tilde{d}-1)(\partial A \partial B)+q r(\partial A \partial F)+r(\tilde{d}-1)(\partial F \partial B)] \\
+ & q\left(\partial_{\alpha} A \partial_{\beta} B+\partial_{\alpha} B \partial_{\beta} A\right)+r\left(\partial_{\alpha} B \partial_{\beta} F+\partial_{\alpha} F \partial_{\beta} B\right) \\
+ & \tilde{d} \Delta B+r \Delta F+\frac{q(q+1)}{2}(\partial A)^{2}+\frac{r(r+1)}{2}(\partial F)^{2}+\frac{\tilde{d}(\tilde{d}-1)}{2}(\partial B)^{2} \\
& \\
& \\
& \\
&
\end{aligned}
$$

For more details see 38. Now one reduces the $(\mu \nu)$-components of (3.1) to the equation

$$
\begin{array}{r}
(q-1) \Delta A+(\tilde{d}+1) \Delta B+r \Delta F \\
+\frac{q(q-1)}{2}(\partial A)^{2}+\frac{r(r+1)}{2}(\partial F)^{2}+\frac{\tilde{d}(\tilde{d}+1)}{2}(\partial B)^{2} \\
+\tilde{d}(q-1)(\partial A \partial B)+r(q-1)(\partial A \partial F)+r \tilde{d}(\partial B \partial F)= \\
-\frac{1}{4}(\partial \phi)^{2}-\frac{h^{2}}{4}(\partial C)^{2} e^{-\alpha \phi-2 q A+2 C}-\frac{k^{2}}{4}(\partial \chi)^{2} e^{2 \tilde{d} B+\beta \phi+2 \chi}
\end{array}
$$

( $\mathrm{nm}$ )-components of (3.1) to the following equation:

$$
\begin{gathered}
q \Delta A+(\tilde{d}+1) \Delta B+(r-1) \Delta F \\
+\frac{q(q+1)}{2}(\partial A)^{2}+\frac{\tilde{d}(\tilde{d}+1)}{2}(\partial B)^{2}+\frac{r(r-1)}{2}(\partial F)^{2} \\
+q \tilde{d}(\partial A \partial B)+q(r-1)(\partial A \partial F)+\tilde{d}(r-1)(\partial B \partial F)= \\
-\frac{1}{4}(\partial \phi)^{2}+\frac{h^{2}}{4}(\partial C)^{2} e^{-\alpha \phi-2 q A+2 C}-\frac{k^{2}}{4}(\partial \chi)^{2} e^{2 \tilde{d} B+\beta \phi+2 \chi}
\end{gathered}
$$


and $(\alpha \beta)$-components to the equation:

$$
\begin{gathered}
-q \partial_{\alpha} \partial_{\beta} A-\tilde{d} \partial_{\alpha} \partial_{\beta} B-r \partial_{\alpha} \partial_{\beta} F \\
-q \partial_{\alpha} A \partial_{\beta} A+\tilde{d} \partial_{\alpha} B \partial_{\beta} B-r \partial_{\alpha} F \partial_{\beta} F+q\left(\partial_{\alpha} A \partial_{\beta} B+\partial_{\alpha} B \partial_{\beta} A\right) \\
+r\left(\partial_{\alpha} B \partial_{\beta} F+\partial_{\alpha} F \partial_{\beta} B\right)+\delta_{\alpha \beta}[q \Delta A+\tilde{d} \Delta B+r \Delta F \\
+\frac{\tilde{d}(\tilde{d}+1)}{2}(\partial A)^{2}+\frac{r(r+1)}{2}(\partial F)^{2} \\
\left.+\frac{\tilde{d}(\tilde{d}-1)}{2}(\partial B)^{2}+q(\tilde{d}-1)(\partial A \partial B)+r(\tilde{d}-1)(\partial F \partial B)+q r(\partial A \partial F)\right] \\
=\frac{1}{2}\left[\partial_{\alpha} \phi \partial_{\beta} \phi-\frac{1}{2} \delta_{\alpha \beta}(\partial \phi)^{2}\right]-\frac{h^{2}}{2} e^{-\alpha \phi-2 q A+2 C}\left[\partial_{\alpha} C \partial_{\beta} C-\frac{\delta_{\alpha \beta}}{2}(\partial C)^{2}\right] \\
-\frac{k^{2}}{2} e^{2 \tilde{d} B+\beta \phi+2 \chi}\left[\partial_{\alpha} \chi \partial_{\beta} \chi-\frac{\delta_{\alpha \beta}}{2}(\partial \chi)^{2}\right]
\end{gathered}
$$

where we use notations $(\partial A \partial B)=\partial_{\alpha} A \partial_{\alpha} B$ and $D=q+r+\tilde{d}+2=d+\tilde{d}+2$.

The equations of motion (3.4) for a part of components of the antisymmetric field are identically satisfied and for the other part they are reduced to a simple equation:

$$
\partial_{\alpha}\left(e^{-\alpha \phi-2 q A+C} \partial_{\alpha} C\right)=0 .
$$

For $\alpha$-components of the antisymmetric field we also have the Bianchi identity:

$$
\partial_{\alpha}\left(e^{\alpha \phi+2 B q+\chi} \partial_{\alpha} \chi\right)=0 .=
$$

The equation of motion for the dilaton has the form

$$
\begin{gathered}
\partial_{\alpha}\left(e^{q A+\tilde{d} B+F r} \partial_{\alpha} \phi\right)-\frac{\alpha h^{2}}{2} e^{-\alpha \phi-q A+q B+r F+2 C}\left(\partial_{\alpha} C\right)^{2} \\
+\frac{\beta k^{2}}{2} e^{\beta \phi+2 \tilde{d} B+2 \chi}\left(\partial_{\alpha} \chi\right)^{2}=0 .
\end{gathered}
$$

We have to solve the system of equations (3.14)-(3.19) for unknown functions $A, B, F, C, \chi, \phi$. We shall express $A, B, F$ and $\phi$ in terms of two functions $C$ and $\chi$. In order to get rid of exponents in (3.14)-(3.19) we impose the following relations:

$$
\begin{gathered}
q A+r F+\tilde{d} B=0, \\
2 \chi+2 \tilde{d} B+\beta \phi=0, \\
2 C-2 q A-\alpha \phi=0 .
\end{gathered}
$$

Under these conditions equations (3.17), (3.18) and (3.19) will have the following forms, respectively,

$$
\begin{gathered}
\partial_{\alpha}\left(e^{-C} \partial_{\alpha} C\right)=0, \quad \partial_{\alpha}\left(e^{-\chi} \partial_{\alpha} \chi\right)=0 \\
\Delta \phi+\frac{\beta k^{2}}{2}\left(\partial_{\alpha} \chi\right)^{2}-\frac{\alpha h^{2}}{2}\left(\partial_{\alpha} C\right)^{2}=0 .
\end{gathered}
$$


One rewrites (3.23) as

$$
\Delta C=(\partial C)^{2}, \quad \Delta \chi=(\partial \chi)^{2} .
$$

Therefore (3.24) will have the form

$$
\Delta \phi+\frac{\beta k^{2}}{2} \Delta \chi-\frac{\alpha h^{2}}{2} \Delta C=0 .
$$

¿From (3.26) it is natural to set

$$
\phi=\phi_{1} C+\phi_{2} \chi,
$$

where

$$
\phi_{1}=\frac{\alpha h^{2}}{2}, \quad \phi_{2}=-\frac{\beta k^{2}}{2} .
$$

¿From equations (3.20), (3.21) and (3.22) it follows that $A, B$ and $F$ can be presented as linear combinations of functions $C$ and $\chi$ :

$$
\begin{aligned}
& A=a_{1} C+a_{2} \chi, \\
& B=b_{1} C+b_{2} \chi, \\
& F=f_{1} C+f_{2} \chi,
\end{aligned}
$$

where

$$
\begin{gathered}
a_{1}=\frac{4-\alpha^{2} h^{2}}{4 q}, \quad a_{2}=\frac{\alpha \beta k^{2}}{4 q}, \\
b_{1}=-\frac{\alpha \beta h^{2}}{4 \tilde{d}}, \quad b_{2}=\frac{\beta^{2} k^{2}-4}{4 \tilde{d}}, \\
f_{1}=\frac{\alpha^{2} h^{2}+\alpha \beta h^{2}-4}{4 r}, \quad f_{2}=\frac{4-\alpha \beta k^{2}-\beta^{2} k^{2}}{4 r} .
\end{gathered}
$$

Let us substitute expressions (3.27), (3.29)-(3.31) for $\phi, A, B, F$ into (3.14)-(3.16). We get relations containing bilinear forms over derivatives on $C$ and $\chi$. We assume that the coefficients in front of these bilinear forms vanish. Then we get the system of twelve quartic equations which is presented and solved in Appendix. The system has a solution only if $\alpha$ and $\beta$ satisfy the relation

$$
\alpha \beta=\frac{2 q \tilde{d}}{q+r+\tilde{d}}
$$

In this case $h$ and $k$ are given by the formulae

$$
\begin{gathered}
h= \pm \sqrt{\frac{4(q+r+\tilde{d})}{\alpha^{2}(q+r+\tilde{d})+2 q(\tilde{d}+r)}}, \\
k= \pm \frac{2 \alpha(q+r+\tilde{d})}{\sqrt{\tilde{d}\left[2 \alpha^{2}(q+r)(q+r+\tilde{d})+4 q^{2} \tilde{d}\right]}}
\end{gathered}
$$

To summarize, the action (1.13) has the solution of the form (1.2) expressed in terms of two harmonic functions $H_{1}$ and $H_{2}$ if the parameters in the action are related by (5.56) and the parameters in the Ansatz $h$ and $k$ are given by (3.36), (3.37). 


\section{Discussion and Conclusion}

Let us discuss different particular cases of the solution (1.2). There is the relation (1.6) between parameters $\alpha$ and $\beta$ in the action (1.1). As a result the action corresponds to the bosonic part of a supergravity theory only in some dimensions.

If $D=4$ and $q=d=1$ then one can take $\alpha=\beta=1$ and the action corresponds to the $S O(4)$ version of $N=4$ supergravity. The solution (1.2) takes the form

$$
d s^{2}=-H_{1}^{-1} H_{2}^{-1} d t^{2}+H_{1} H_{2} d x^{\alpha} d x^{\alpha}
$$

This supersymmetric solution has been obtained in 21.

If $\alpha=\beta$ and $q=\tilde{d}$ then one has the solution

$$
d s^{2}=H_{1}^{\frac{2}{D-2}} H_{2}^{\frac{2(D-q-2)}{q(D-2)}}\left[\left(H_{1} H_{2}\right)^{-\frac{2}{q}} \eta_{\mu \nu} d y^{\mu} d y^{\nu}+H_{2}^{-\frac{2}{q}} d z^{m} d z^{m}+d x^{\alpha} d x^{\alpha}\right]
$$

This solution was obtained in [38]. It contains as a particular case for $d=10, q=2$ the known solution [30, 8, 36]

$$
\begin{gathered}
d s^{2}=H_{1}^{-\frac{3}{4}} H_{2}^{-\frac{1}{4}}\left(-d y_{0}^{2}+d y_{1}^{2}\right) \\
+H_{1}^{\frac{1}{4}} H_{2}^{-\frac{1}{4}}\left(d z_{2}^{2}+d z_{3}^{2}+d z_{4}^{2}+d z_{5}^{2}\right)+H_{1}^{\frac{1}{4}} H_{2}^{\frac{3}{4}}\left(d x_{6}^{2}+d x_{7}^{2}+d x_{8}^{2}+d x_{9}^{2}\right) .
\end{gathered}
$$

This solution has been used in the D-brane derivation of the black hole entropy [7, 8]. Note however that the solution (4.40) corresponds to the action (1.1) with the 3-form $F_{3}$ and the 7 -form $G_{7}$.

To conclude, a rather general three-block solution of the action (1.1) has been constructed. It contains as particular cases many known solutions. However the solution is not general enough to include some known multi-block solutions. Further work is needed to understand better the structure hidden behind the multi-block p-brane solutions.

\section{Acknowlegments}

This work is supported by an operating grant from the Natural Sciences and Engineering Research Council of Canada. I.A. and I.V. thank the Department of Physics for the kind hospitality during their stay at Simon Fraser University. I.V. is partially supported by the RFFI grant 9600312.

\section{Appendix}

We obtain the following system of algebraic equations

$$
\begin{gathered}
-a_{1}+b_{1}+\frac{q(q-1)}{2} a_{1}^{2}+\frac{r(r+1)}{2} f_{1}^{2}+\frac{\tilde{d}(\tilde{d}+1)}{2} b_{1}^{2} \\
+\tilde{d}(q-1) a_{1} b_{1}+r(q-1) a_{1} f_{1}+r \tilde{d} f_{1} b_{1}+\frac{\phi_{1}^{2}}{4}+\frac{h^{2}}{4}=0 ; \\
-a_{2}+b_{2}+\frac{q(q-1)}{2} a_{2}^{2}+\frac{r(r+1)}{2} f_{2}^{2}+\frac{\tilde{d}(\tilde{d}+1)}{2} b_{2}^{2}
\end{gathered}
$$




$$
\begin{aligned}
& +\tilde{d}(q-1) a_{2} b_{2}+r(q-1) a_{2} f_{2}+r \tilde{d} b_{2} f_{2}+\frac{\phi_{2}^{2}}{4}+\frac{k^{2}}{4}=0 \\
& q(q-1) a_{1} a_{2}+r(r+1) f_{1} f_{2}+\tilde{d}(\tilde{d}+1) b_{1} b_{2} \\
& +\tilde{d}(q-1)\left(a_{1} b_{2}+a_{2} b_{1}\right)+r(q-1)\left(a_{2} f_{1}+a_{1} f_{2}\right)+r \tilde{d}\left(f_{1} b_{2}+f_{2} b_{1}\right)+\frac{\phi_{1} \phi_{2}}{2}=0 \\
& -f_{1}+b_{1}+\frac{q(q+1)}{2} a_{1}^{2}+\frac{r(r-1)}{2} f_{1}^{2}+\frac{\tilde{d}(\tilde{d}+1)}{2} b_{1}^{2} \\
& +q \tilde{d} a_{1} b_{1}+q(r-1) a_{1} f_{1}+\tilde{d}(r-1) f_{1} b_{1}+\frac{\phi_{1}^{2}}{4}-\frac{h^{2}}{4}=0 \\
& -f_{2}+b_{2}+\frac{q(q+1)}{2} a_{2}^{2}+\frac{r(r-1)}{2} f_{2}^{2}+\frac{\tilde{d}(\tilde{d}+1)}{2} b_{2}^{2}+ \\
& +q \tilde{d} a_{2} b_{2}+q(r-1) a_{2} f_{2}+\tilde{d}(r-1) b_{2} f_{2}+\frac{\phi_{2}^{2}}{4}+\frac{k^{2}}{4}=0 \\
& q(q+1) a_{1} a_{2}+r(r-1) f_{1} f_{2}+\tilde{d}(\tilde{d}+1) b_{1} b_{2} \\
& +q \tilde{d}\left(a_{1} b_{2}+a_{2} b_{1}\right)+q(r-1)\left(a_{2} f_{1}+a_{1} f_{2}\right)+\tilde{d}(r-1)\left(f_{1} b_{2}+f_{2} b_{1}\right)+\frac{\phi_{1} \phi_{2}}{2}=0 \\
& -q a_{1}^{2}-r f_{1}^{2}+\tilde{d} b_{1}^{2}+ \\
& +2 q a_{1} b_{1}+2 r f_{1} b_{1}-\frac{\phi_{1}^{2}}{2}+\frac{h^{2}}{2}=0 \\
& -q a_{2}^{2}-r f_{2}^{2}+\tilde{d} b_{2}^{2} \\
& +2 q a_{2} b_{2}+2 r b_{2} f_{2}-\frac{\phi_{2}^{2}}{2}+\frac{k^{2}}{2}=0 \\
& -q a_{1} a_{2}-r f_{1} f_{2}+\tilde{d} b_{1} b_{2} \\
& +q\left(a_{1} b_{2}+a_{2} b_{1}\right)+r\left(f_{1} b_{2}+f_{2} b_{1}\right)-\frac{\phi_{1} \phi_{2}}{2}=0 ; \\
& \frac{q(q+1)}{2} a_{1}^{2}+\frac{r(r+1)}{2} f_{1}^{2}+\frac{\tilde{d}(\tilde{d}-1)}{2} b_{1}^{2} \\
& +q(\tilde{d}-1) a_{1} b_{1}+r(\tilde{d}-1) b_{1} f_{1}+r q f_{1} a_{1}+\frac{\phi_{1}^{2}}{4}-\frac{h^{2}}{4}=0 ;
\end{aligned}
$$




$$
\begin{gathered}
\frac{q(q+1)}{2} a_{2}^{2}+\frac{r(r+1)}{2} f_{2}^{2}+\frac{\tilde{d}(\tilde{d}-1)}{2} b_{2}^{2} \\
+q(\tilde{d}-1) a_{2} b_{2}+r(\tilde{d}-1) a_{2} f_{2}+r q b_{2} f_{2}+\frac{\phi_{2}^{2}}{4}-\frac{k^{2}}{4}=0 ; \\
q(q+1) a_{1} a_{2}+r(r+1) f_{1} f_{2}+\tilde{d}(\tilde{d}-1) b_{1} b_{2}+q(\tilde{d}-1)\left(a_{1} b_{2}+a_{2} b_{1}\right) \\
+r(\tilde{d}-1)\left(b_{2} f_{1}+b_{1} f_{2}\right)+r q\left(f_{1} a_{2}+f_{2} a_{1}\right)+\frac{\phi_{1} \phi_{2}}{2}=0 .
\end{gathered}
$$

Now let us discuss the system of eq. (5.41)-(5.52). The action depends on the parameters $D, \alpha, \beta, q, \tilde{d}$. We have used the parameter $r$ instead of $D$ which is defined from

$$
q+r+\tilde{d}+2=D
$$

Therefore our action (1.13) depends on five parameters $r, \alpha, \beta, q, \tilde{d}$ We have also two parameters $h$ and $k$ in our Ansatz (3.9),(3.10). We substitute expressions (3.27),(3.28), (3.32)-(3.34) into (5.41)-(5.52). Then we get the system of twelve quartic equations for seven unknown variables $r, \alpha, \beta, q, \tilde{d}, h$ and $k$. Using Maple $\mathrm{V}$ we found the solution of the system of equations (5.41)-(5.52). The solution has the form

$$
\begin{gathered}
k=\frac{\sqrt{2 q(r+\tilde{d}) h^{2}-4(q+r+\tilde{d})}}{\sqrt{\tilde{d}\left(q r h^{2}-2(q+r)\right)}}, \\
\alpha=\frac{\sqrt{4(q+r+\tilde{d})-2 q(r+\tilde{d}) h^{2}}}{h \sqrt{q+r+\tilde{d}}} \\
\beta=\frac{2 q \tilde{d} h}{\sqrt{(q+r+\tilde{d})\left[4(q+r+\tilde{d})-2 q(r+\tilde{d}) h^{2}\right]}}
\end{gathered}
$$

where $r, q, \tilde{d}, h$ are arbitrary. Let us rewrite the solution in terms of parameters in the action. ¿From equations (5.54) and (5.55) follows that $h$ can be found from equations (5.41)-(5.52) only if $\alpha$ and $\beta$ are subjected to the relation

$$
\alpha \beta=\frac{2 q \tilde{d}}{q+r+\tilde{d}}
$$

In this case $h^{2}$ and $k^{2}$ are given by the formulae

$$
\begin{gathered}
h^{2}=\frac{4(q+r+\tilde{d})}{\alpha^{2}(q+r+\tilde{d})+2 q(\tilde{d}+r)}, \\
k^{2}=\frac{2 \alpha^{2}(q+r+\tilde{d})^{2}}{\tilde{d}\left[\alpha^{2}(q+r)(q+r+\tilde{d})+2 q^{2} \tilde{d}\right]}
\end{gathered}
$$




\section{References}

[1] A.Font, L.Ibanez, D.Lust and F.Quevedo, Phys.Let.B249(1990)35

[2] A.Sen, Int.J.Mod.Phys.A8(1993)2023

[3] C.Hull and P.Townsend, Nucl.Phys.B438(1995)109

[4] E.Witten, Nucl.Phys.B443(1995)85

[5] J.H.Schwarz, hep-th/9607201

[6] C.Vafa, hep-th/9602022

[7] A.Strominger and C.Vafa, hep-th/9601029

[8] C.Callan and J.Maldacena, hep-th/9602043

[9] J.Breckenridge, R.Myers, A.Peet and C.Vafa, hep-th/9602065

[10] J.Maldacena and A.Strominger, hep-th/9603060

[11] C.Johnson, R.Khuri and R.Myers, hep-th/9603061

[12] G.Horowitz, J.Maldacena and A.Strominger, hep-th/9603109

[13] G.Horowitz, gr-qc/9604051

[14] J.Polchinski, hep-th/9511026

[15] J.Polchinski,S.Chaudhuri and C.Johnson,hep-th/960252

[16] A.Dubholkar, G.W.Gibbons, J.A.Harvey, F.Ruiz Ruiz, Nucl.Phys. 340 (1990) 33.

[17] C.G.Callan, J.A.Harvey, A.Strominger, Nucl.Phys. 359 (1991) 611; 367 (1991) 60.

[18] M.J.Duff and K.S.Stelle, Phys.Lett.253(1991) 113.

[19] G.T.Horowitz and A.Strominger, Nucl.Phys.360(1991) 197.

[20] R.Guven, Phys.Lett.276(1992) 49, Phys.Lett.212(1988) 277.

[21] R.Kallosh, A.Linde, T.Ortin, A.Peet and A.van Proeyen, Phys.Rev. D46 (1992) 5278 .

[22] H.Lu, C.N.Pope, E.Sergin and K.Stelle, hep-th/9508042.

[23] M.J.Duff, R.R.Khuri and J.X.Lu, Phys.Rep. 259 (1995) 213.

[24] G.W.Gibbons, G.T.Horowitz and P.K.Townsend, Class. Quant. Grav.12 (1995) 297.

[25] J.M.Schwarz, Phys.Lett.B360(1995)13

[26] H.Lu and C.N.Pope, hep-th/9512153; hep-th/9512012

[27] J.Rahmfeld, hep-th/9512089 
[28] M.Cvetic and D.Youm, hep-th/9510098

[29] M.Cvetic and A.A.Tseytlin, hep-th/9512031

[30] A.A.Tseytlin, hep-th/9601177

[31] I.R.Klebanov and A.A.Tseytlin, hep-th/9604166

[32] G.Papadopoulos and P.K.Townsend, hep-th/9603087

[33] K.Behrndt, E.Bergshoeff and B.Janssen, hep-th/9604168

[34] K.Behrndt and R.Kallosh, Phys.Rev.D 53 (1996) R589.

[35] A.A.Tseytlin, hep-th/9604035

[36] M.Cvetic and C.M.Hull, hep-th/9606193

[37] E.Bergshoeff, R.Kallosh, T.Ortin, hep-th/9605059

[38] A.Volovich, hep-th/9608095 